\title{
Comamonas kerstersii
}

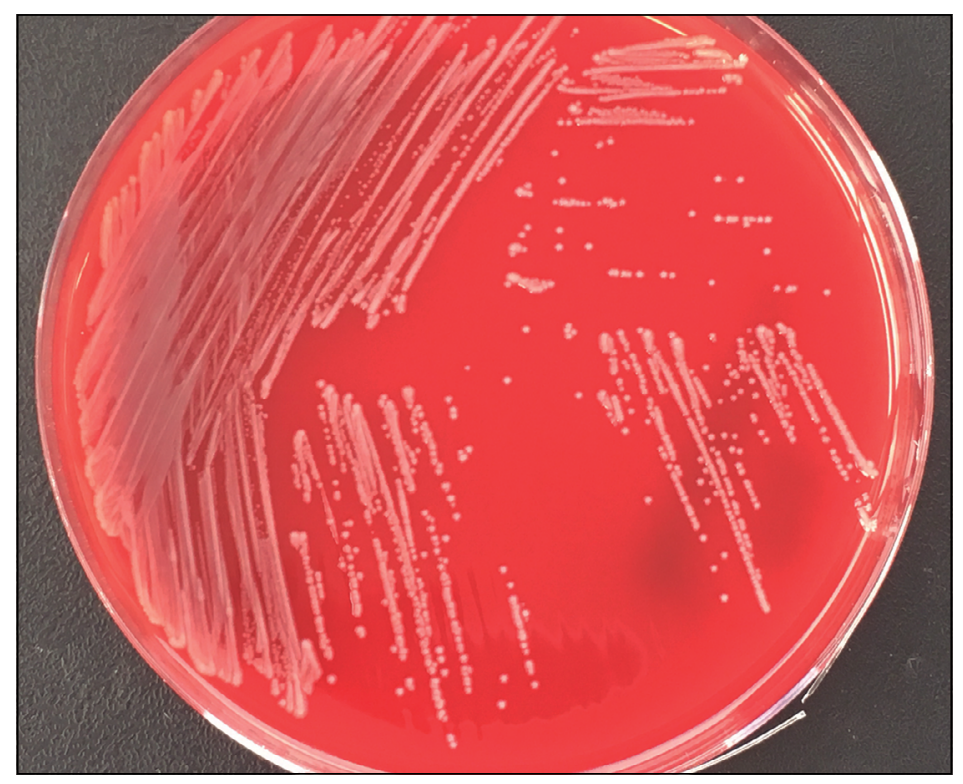

Figura 1. Colonias de Comamonas kerstersii en agar sangre ovina .

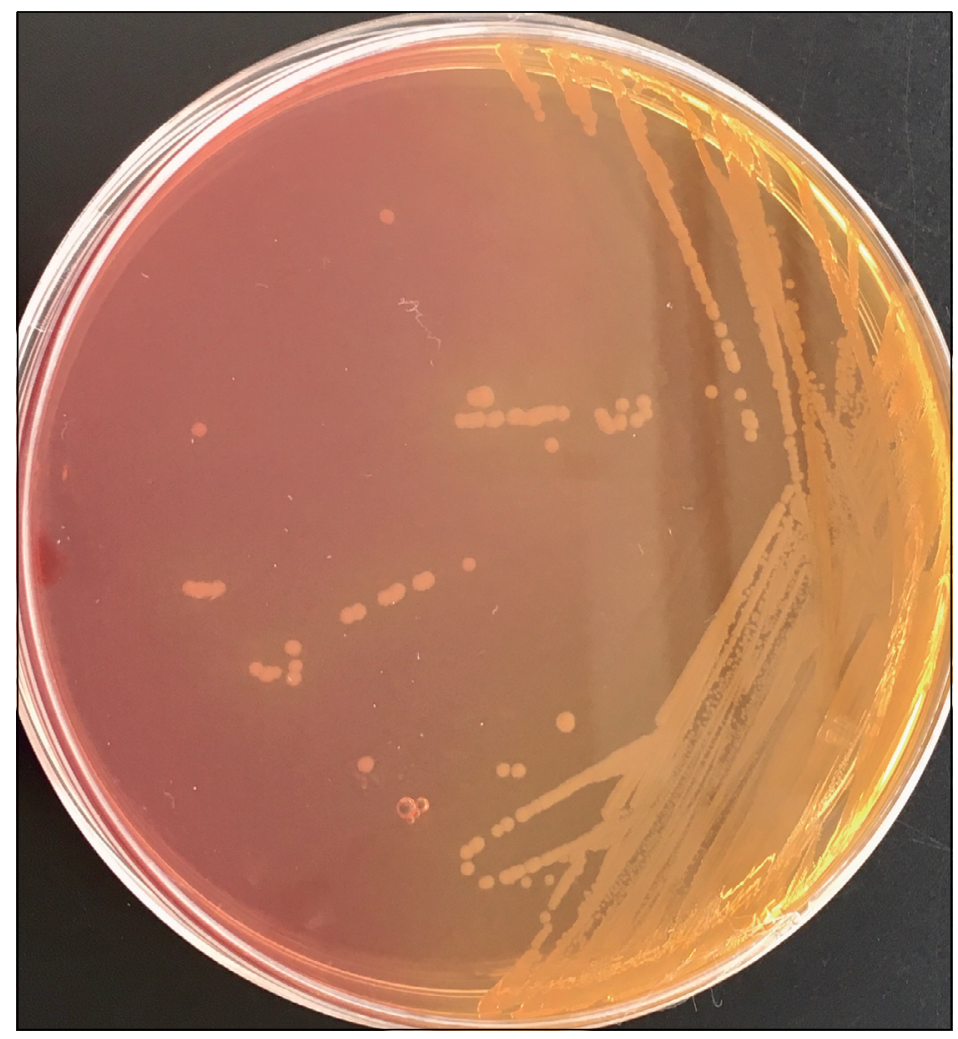

Figura 2. Colonias de Comamonas kerstersii en agar MacConkey. 


\section{Comamonas kerstersii}

El género Comamonas fue originalmente identificado en 1985 e incluía una sola especie: Comamonas terrigena. Poco después, Pseudomonas acidovorans y Pseudomonas testosteroni se reclasificaron como miembros del género Comamonas. El primero fue; sin embargo, nuevamente reclasificado como Delftia acidovorans. Comamonas terrigena incluyeron tres grupos de cepas de aislados clínicos humanos, que ahora se conocen como C. terrigena, C. aquatica y C. kerstersii. El género Comamonas, actualmente totaliza 16 especies, que son principalmente de origen ambiental.

Se trata de bacilos gramnegativos rectos a ligeramente curvos que se presentan solos o en pares. Son no fermentadores y crecen bien en los medios de cultivo utilizados habitualmente en el laboratorio de microbiología clínica, incluyendo el agar MacConkey. Presentan reacción positiva para catalasa y oxidasa. Son móviles a través de un mechón de flagelos polares. Todas las especies de Comamonas, incluyendo C. kerstersii, reducen nitratos a nitritos. Wauters y cols. plantean un esquema de pruebas bioquímicas y crecimiento a diferentes temperaturas para la identificación de especie. Pero esta diferenciación fenotípica es difícil, con pruebas no siempre al alcance de los laboratorios clínicos. Como resultado, los aislados generalmente se informan como Comamonas spp. Asimismo, C. kerstersii no esta incluída en la base de datos de diferentes sistemas comerciales de identificación fenotípica. Con la incorporación de la identificación a través de Matrix-Assisted Laser Desorption Ionización-Time of Flight (MALDI-TOF) se ha logrado la correcta identificación de C. kerstersii, diferenciándola correctamente de las demás especies de Comamonas.

La mayoría de las infecciones reportadas corresponden a infecciones intraabdominales donde C. kerstersii se presenta, frecuentemente, asociado a otros agentes. Habitualmente, es susceptible a la mayoría de los antimicrobianos ensayados en bacilos gramnegativos no fermentadores. Los casos descritos en la literatura médica han tenido una evolución favorable.

\section{Referencias bibliográficas}

1.- Lipuma JJ, Currie BJ, Peacock SJ, Vandamme PAR. Burkholderia, Stenotrophomonas, Ralstonia, Cupriavidus, Pandoraea, Brevundimonas, Comamonas, Delftia, and Acidovorax. En: Jorgensen JH, Pfaller MA. Manual of Clinical Microbiology: ASM Press. 2015: 791-812.

2.- Bacilos Gram negativos no fermentadores. En: Procop GW, Church DL, Hall GS, Janda WH, Koneman EW, Schreckenberger PC, et al. Koneman Diagnóstico Microbiólogico. Philadelphia: Walters Kluwer; 2017: 316-431.

3.- Wauters G, De Baere T, Willems A, Falsen E, Vaneechoutte M. Description of Comamonas aquatica comb. nov. and Comamonas kerstersii sp. nov. for two subgroups of Comamonas terrigena and emended description of Comamonas terrigena. Int J Syst Evol Microbiol 2003; 53: 859-62. doi: 10.1099/ijs.0.02450-0.

4.- Almuzara MN, Cittadini R, Vera Ocampo C, Bakai R,Traglia G, Ramirez MS, et al. Intraabdominal Infections due to Comamonas kerstersii. J Clin Microbiol 2013; 51: 1998-2000. doi: 10.1128/JCM.00659-13.

5.- Bizzini A, Durussel C, Bille J, Greub G, Prod'hom G. Performance of matrix-assisted laser desorption ionization-time of flight mass spectrometry for identification of bacterial strains routinely isolated in a clinical microbiology laboratory. J Clin Microbiol 2010; 48: 1549-54. doi: 10.1128/JCM.01794-09.

Rosario Palacio ${ }^{1}$, Cecilia Cornejo ${ }^{1}$ y Verónica Seija ${ }^{1}$ ${ }^{1}$ Laboratorio de Patología Clínica. Hospital de Clínicas. Facultad de Medicina. Universidad de la República. Montevideo Uruguay.

Correspondencia a: Verónica Seija veronicaseija@hc.edu.uy 